\title{
Cancer immunology, inflammation, and tolerance: an introduction
}

\author{
Stefan Dirnhofer ${ }^{1} \cdot$ Alfred Zippelius $^{2}$
}

Published online: 23 February 2019

(C) Springer-Verlag GmbH Germany, part of Springer Nature 2019

In the last decade, groundbreaking achievements have been obtained in the field of oncology. These have been fueled by the strong belief that a better knowledge of genetic or molecular mechanisms sustaining cancer growth could be key for more efficacious treatments. The results of a number of clinical trials showed that only a minority of patients benefited from targeted therapies, thus underlying that something else was missing in the scenario composed by molecular signatures, trunk and branch mutations, and conventional histopathological features to expand clinical results to most patients.

Clinical advances in immunotherapy were built on four main discoveries: (i) the biological understanding that cancer immunoediting, a key mechanism through which tumor cells overcome the immune response, paradoxically promotes cancer development and growth; (ii) the identification of the remarkable genetic and immunologic heterogeneity of human tumors; (iii) the preclinical evidence that efficient anticancer strategies should focus on the simultaneous targeting of multiple pathways; (iv) the appreciation from ex vivo clinical data of the concept of tumor microenvironment (TME), namely the existence, along with tumor cells, of non-tumor components that heavily affect tumor growth and survival. In this year's Annual Review Issue, we have decided to focus on preclinical and clinical advances through which immunotherapy came of age. To cover such a large and diverse series of topics, we called upon a group of experts who are leading authorities in their respective fields to produce cutting-edge review articles.

In the first paper of the series, Tuccitto et al. (https://doi.org/ $10.1007 / \mathrm{s} 00428-018-2477-z)$ describe the complex network of TME-driving forces. The authors report the molecular features

Stefan Dirnhofer

Stefan.dirnhofer@usb.ch

1 Institute of Medical Genetics and Pathology, University Hospital Basel, Schönbeinstrasse 40, 4031 Basel, Switzerland

2 Medical Oncology, University Hospital Basel \& Laboratory Cancer Immunology, Department Biomedicine, University Basel, Basel, Switzerland of TME and antitumor adaptive immunity, where metabolic changes contribute to cancer dissemination by impairing $T$ cell infiltration and favoring the recruitment and activation of regulatory cells. The authors underline recent findings that oncogenic pathways and specific mutations in tumor cells dictate the nature and the function of the immune infiltrate. The translational relevance of these findings is underlined by their potential for the discovery of novel biomarkers and therapeutic targets to improve treatment efficacy in cancer.

Two papers by Ottaviano et al. (https://doi.org/10.1007/ s00428-019-02538-4) and Mandalà et al. (https://doi.org/10. $1007 / \mathrm{s} 00428-018-2506-y)$ point to the recent advances in cancer immunotherapy describing the unprecedented success in the treatment of melanoma by using anti-CTLA4, anti-PD1 antibodies, and their combination. In particular, the paper by Ottaviano et al. reports the state of the art in cancer immunotherapy, while Mandalà and Rutkowski, by underlining the complexity of immune system and the multiple homeostatic mechanisms which control immune responses, show that combinatorial strategies are eagerly needed in melanoma therapy. The authors underscore that a rational selection of immunotherapy combinations should be rigorously biomarkerguided, including cancer immunogram, PD-L1 expression, interferon gene expression signature, mutational burden, tumor infiltration by CD8+ T cells, intratumoral $\mathrm{T}$ cell exhaustion, and microbiota composition.

The immune checkpoint blockade, a major breakthrough in cancer treatment, is one current option for the treatment of multiple tumor types. Adoptive cell therapy (ACT) with tumor-infiltrating lymphocytes (TIL) or gene-modified T cells expressing novel $\mathrm{T}$ cell receptors or chimeric antigen receptors (CAR) is an additional strategy to strengthen the antitumor immune system program. This ACT topic, covered by Rohaan et al. (https://doi.org/10.1007/s00428-0182484-0), has shown promising results in various tumor types, and multiple clinical trials are being conducted worldwide to further optimize this treatment modality. The authors provide an overview of the developments in $\mathrm{ACT}$, the associated toxicity, and the future potential of $\mathrm{ACT}$ in cancer treatment. 
The expansion of research on the tumor immune microenvironment, the successes and dissemination of immunotherapies to treat cancer, and the facilitated access to large "-omic databases" have boosted the development of novel powerful immune-based prognostic and theranostic biomarkers. Although they often correlate with histopathologic characteristics and TNM staging, in many instances, they are independently associated with, and potentially superior predictors of, the patient's prognosis and response to immunotherapies. The review by Giraldo et al. (https://doi.org/10.1007/s00428-01802517-1) links the composition of the tumor immune microenvironment with the patient's prognosis and the response to checkpoint blockade in solid cancer, integrating these findings with novel technologies to improve their predictive potential.

Savic -Prince and Bubendorf (https://doi.org/10.1007/ s00428-018-2445-7) focus on the complex biological background of PD-L1 as predictive cancer biomarker, summarizing relevant clinical trials in NSCLC and illustrating the origin of different PD-L1 expression cutoffs and scorings. The authors also address important issues for the understanding of PD-L1 testing including the analytical comparability of the different clinical trial-validated PD-L1 immunohistochemistry assays, the potential of laboratory-developed tests, and an overview of the different scoring algorithms.

The advent of new cancer immunotherapy approaches and emerging combination therapies has led to new challenges, including starting and stopping rules for immunotherapy, the management of immune-related adverse events, and logistic issues for the production of cellular therapies and viral delivery vectors. The review by Läubli et al. (https://doi.org/10. 1007/s00428-018-2435-9) supports the need of a highly interdisciplinary immune tumor board for the optimal management of cancer patients treated with immunotherapy and provide an outlook on how such a platform could be used in the future to discuss rational and personalized combination therapies, and how to improve the management of side effects deriving from immunotherapy.
The chromosomal region 9p24 harbors several genes implicated in immune checkpoint inhibition therapy, targeted signaling inhibition, and epigenetic modulation. In their paper, Menter and Tzankov (https://doi.org/10.1007/s00428-0182438-6) describe the role of genes located on chromosome 9p24 and related proteins in lymphoma subtypes with a special focus on PD-L1 and PD-L2. Specific attention is paid to the amplification of PD-L1 as a hallmark of both classical Hodgkin and primary mediastinal B cell lymphoma, and JAK2, which is point-mutated in myeloproliferative neoplasms and other myeloid malignancies, and rearranged in PCM1-JAK2-positive myeloid/lymphoid neoplasms with eosinophila. The presence of KDM4C/JMJD2C, which is relevant for oncogenesis, further underlines the key role of this locus.

In the final paper of the series, Koelzer et al. (https://doi.org/ 10.1007/s00428-018-2485-z) critically assess computational approaches supporting the development of a standardized methodology in the assessment of immune-oncology biomarkers, such as PD-L1 and immune cell infiltrates. Immunoprofiling through spatial analysis of tumor immune cell interactions and multiplexing technologies as a predictor of patient response to cancer treatment are discussed. The authors provide an overview on machine learning and artificial intelligence tools and illustrate fields of application in immune-oncology, such as pattern-recognition in large and complex datasets and deep learning approaches for survival analysis.

In conclusion, we hope that this Annual Review Issue has assembled a collection of review articles which underscore the importance of the multidisciplinary team of pathologists, immunologists, oncologists, and bioinformaticists to identify personalized biomarker profiles, and attracts the interest of both scientists and clinicians dedicated to cancer immunotherapy and the optimization of drug combination therapies.

Publisher's note Springer Nature remains neutral with regard to jurisdictional claims in published maps and institutional affiliations. 